\title{
The control of naturally ventilated buildings subject to wind and buoyancy
}

\author{
By BEN LISHMAN AND ANDREW W. WOODS \\ BP Institute, Madingley Rise, Madingley Road, Cambridge CB3 0EZ, UK
}

(Received 5 June 2005 and in revised form 1 December 2005)

The competition between wind and buoyancy forces acting on a naturally ventilated building with one upwind and one downwind opening and a distributed heat source can lead to multiple steady states. This study considers the impact of a second downwind opening on the existence of these multiple steady states. It is found that if this new opening exceeds a critical area, then the multiple steady states are eliminated, and the transition from buoyancy to wind dominated flow is continuous. This critical area is shown to be a function of the relative heights of the three windows. The predictions of the model are in accord with new laboratory experiments, and the implications for building design are discussed.

\section{Introduction}

With rising global energy consumption, continued growth of primary energy supply becomes progressively more challenging, and there is a need for substantial improvements in energy efficiency. For example, buildings account for $47 \%$ of energy consumption in the United Kingdom (Department of Trade and Industry 2003). However, there has been a trend towards increased control of the interior environment of buildings, and this has led to widespread use of air-conditioning within public buildings. Natural ventilation, as an alternative to such air conditioning, often provides an opportunity for significant reductions in the energy consumption. However, in order for natural ventilation to be a credible alternative, it should provide an environment of comparable comfort to that associated with a purely mechanical ventilation system. This is a substantial challenge, since the controls available for natural ventilation systems are simple, and therefore we require a much deeper understanding of the flow patterns within a building (Linden 1999).

In building up understanding of natural ventilation flows, with the ultimate goal of developing control systems, the flow within simplified model buildings has been examined. In a large open-plan space, with one upper- and one lower-level opening, it has been shown that the nonlinear interaction of wind-induced pressure gradients and the buoyancy forces associated with internal heating can lead to multiple flow regimes (Li et al. 2000; Hunt \& Linden 2000; Gladstone \& Woods 2001; Hunt \& Linden 2004). The existence of such multiple nonlinear steady states, corresponding to a wind-dominated flow and a buoyancy-dominated flow, may be challenging for the design of a control system.

However, many buildings are in fact of more complex geometry, and include three or more openings to the exterior. Here we show that this may lead to a fundamental difference in the flow regime as compared to a building with just two openings. In particular, we show that in a building with three openings, if the upper downwind 

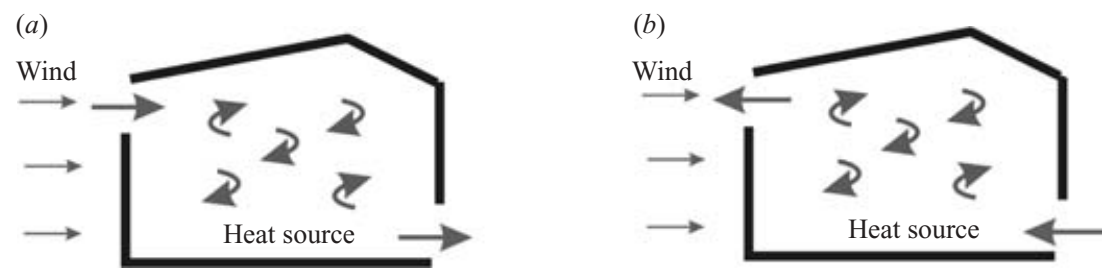

FiguRE 1. With two openings, two regimes are possible: $(a)$ a wind-dominated regime, and (b) a buoyancy-dominated regime.

window is sufficiently large, then as the internal heat load increases there may be a smooth adjustment from the wind-dominated to the buoyancy-dominated flow regime, with no intermediate regime in which there are multiple steady states.

Before launching into a description of our quantitative modelling and laboratory experiments, it is useful to contrast how wind and buoyancy forces compete in a simplified open-plan building with two and with three openings. In order to elucidate some of the basic principles, we examine the case of a building with a uniform heat load on the floor which produces a well-mixed interior. Earlier work has examined the case of point sources of heating, which can generate internal stratification in the buoyancy-dominated flow regime (see Hunt \& Linden 2000, 2004), although the mixing ventilation regime associated with the wind-driven flow is analogous to that produced with a distributed source of heating of the same heat flux. Real buildings are likely to have a variety of heat sources which lead to different degrees of vertical stratification within the space; the present model of uniform areal heat load represents one end of this spectrum which allows us to explore some of the principles that control transitions in building scale flow.

First, we consider a building with a distributed heat source on the floor and two openings, one at high level upwind and one at low level downwind, as shown in schematic form in figure 1. Both windows are assumed to be of relatively small vertical extent. With a very small wind speed, buoyancy forces will dominate, leading to an upward displacement flow through the building, with inflow at the base and outflow through the upper opening. In contrast, with a strong wind, we expect the wind pressure gradient to dominate, with the flow entering at high level, upwind of the building, and migrating to the lower downwind vent. However, as the wind slowly increases from small to large values, there is no continuous path through the steadystate solutions from the wind-driven regime to the buoyancy-driven regime. This is readily seen by recognizing that if there were a smooth transition, then there would need to be a critical wind speed at which there is no net flow through either opening. However, in this case, the continuing heat load would tend to heat up the space so that the buoyancy force exceeds the wind-induced pressure, and the buoyancy-driven flow would resume. We deduce that there is no smooth transition. Instead, as explained by Gladstone \& Woods (2001), we expect that a stable slow and high-temperature buoyancy-driven mode, and a stable fast and low-temperature wind-driven mode can both develop for a range of intermediate conditions. If the flow adjusts from one steady region to the other, there will be a finite change in the ventilation rate and the interior temperature. We refer to this transition as a discontinuous change in the steady-state regime.

In contrast to this discontinuous transition in flow with only two openings, in this paper we show that if there is a further opening, of similar size, at a high level on the downwind face, then the flow may adjust continuously through a series of 
(a)

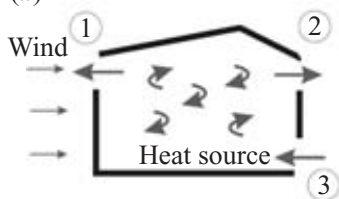

(b)

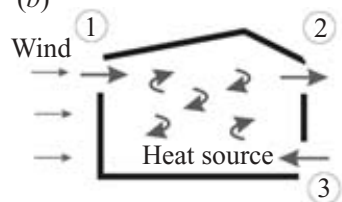

(c)

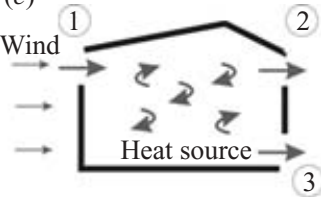

FIGURE 2. With three openings, three regimes are possible: $(a)$ a buoyancy dominated regime with inflow through the low downwind vent, and outflow through both upper vents; $(b)$ an intermediate regime with inflow through the upwind and low downwind vents, and outflow through the upper downwind vent; and $(c)$ a wind-dominated regime with inflow through the upwind vent and outflow through the downwind vents.

steady-state solutions from the wind-driven to the buoyancy-driven flow. To illustrate this difference, we consider in detail the inclusion of a new high-level opening on the downwind face of the building (figure 2). The transition from buoyancy- to wind-driven flow may be understood by considering in detail the flow through the three vents as the wind speed increases from small to large values. At small wind speeds, the heating within the space leads to a pure upward displacement mode of ventilation, with inflow at the base and outflow through both high-level vents (figure $2 a$ ). As the wind increases, the flow through the high-level upwind vent falls to zero and then reverses, while the flow through the two downwind vents remain of finite value, thereby venting the heat load from the space (figure $2 b$ ). As the wind increases further, the flow through the low-level downwind vent reverses to become an outflow; at this second critical wind speed, there is still a finite in/out flow through the two high-level vents which acts to ventilate the heat generated within the space (figure $2 c$ ).

Real buildings are likely to have three or more openings, and so for a given heat load and wind speed, it should be possible to ensure that for each pair of values of wind speed and flow, there is a unique flow regime. However, with two openings, we have seen that there is a set of conditions for which the flow is non-unique, and so the transition between flow regimes is discontinuous. By continuity, it follows that in a building with three openings, but in which the upper downwind opening is rather small, there may be a range of conditions for which the flow is non-unique and hence the transitions in flow regime may again be discontinuous.

The main purpose of this paper is to identify the key factors which control whether in a building with three openings, the flow is uniquely determined in terms of the wind speed and heat load. This situation leads to much simpler control of the natural ventilation, and smooth rather than discontinuous transitions in flow regime.

In $\S 2$, we develop a model of the natural ventilation within an open-plan building with three openings (figure 2), in which there is a distributed heating system at low level. We examine the controls on the flow regime and flow rate as both the heat load and the size of the high-level downwind opening change for a given size of the other openings. We compare the predictions of the model with a series of new analogue laboratory experiments in which a heated rectangular tank of water, with three ventilation openings, is placed in a steady background flow within a flume. We then explore how the size and height of the upper downwind opening affects the uniqueness of the flow as the heat load and wind speed vary. Using these results, we map out a bifurcation diagram of the different flow regimes as a function of opening area and height of the opening, and we discuss the implications of these results for design and control of naturally ventilated buildings. 


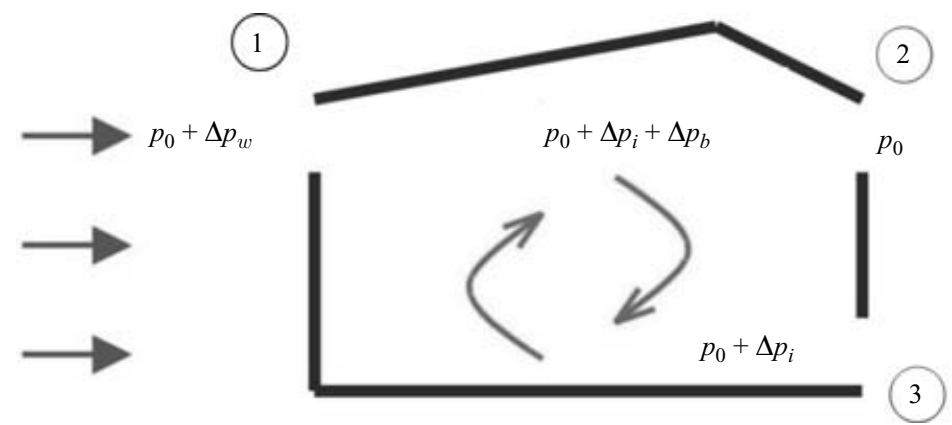

FIGURE 3. Pressures acting on the building. We assume a background pressure $p_{0}$, and note that three further pressures need to be considered: the wind forcing $\Delta p_{w}$, which is positive from exterior to interior at the upper upwind opening, the hydrostatic pressure difference between the exterior cold air and interior warm air, $\Delta p_{b}$, which is positive from interior to exterior at the upper openings, and the pressure difference between the lower interior and exterior, $\Delta p_{i}$, which is positive from interior to exterior at all openings. The sign of $\Delta p_{i}$ varies with the flow regime.

\section{Modelling approach}

We develop a simplified model of the overall ventilation flow and heat budget within a model building. Figure 3 illustrates the geometry of the simplified model building we consider. We assume the building has high-level openings at the same height on the upwind and downwind faces, and a further low-level opening on the downwind face (below the other two openings). In $\S 5$, we will generalize the analysis to allow the height of the upper downwind opening to differ from that of the upper upwind opening. We use the suffices 1, 2 and 3 to represent the upper upwind opening, upper downwind opening and lower downwind opening, respectively (see figure 3). For simplicity, we consider the case when the areas of the upper upwind and lower downwind openings are the same, i.e. $A_{1}=A_{3}$.

We assume that the underfloor distributed heating leads to high-Rayleigh-number convection and hence a well-mixed interior (cf. Gladstone \& Woods 2001). We also assume that each opening is of sufficiently small vertical extent compared to the depth of the room that the flow is purely in- or outflow, as controlled by the pressure difference across the mid-height of that opening (cf. Linden, Lane-Serff \& Smeed 1990). This implies that the volume flow through each opening is (see Linden et al. 1990):

$$
Q_{x}=c^{*} A_{x} \sqrt{\frac{\Delta p_{x}}{\rho}},
$$

where $Q_{x}$ is the volume flow through an opening, $A_{x}$ is the area of that opening, $\Delta p_{x}$ is the pressure difference across the opening, $c^{*}$ is a loss coefficient and $\rho$ is the fluid density. For convenience, we assume that the loss coefficient $c^{*}$ is the same for each opening of our model building.

The pressure difference between the upwind and downwind faces of the building associated with the wind is taken to have the constant value $\Delta p_{w}$. We use $\Delta p_{b}$ to represent the hydrostatic pressure difference between the exterior cold air and interior warm air, measured between the mid-heights of the high-level and low-level openings, a vertical distance $h$ apart. $\Delta p_{b}$ is given by

$$
\Delta p_{b}=\rho g^{\prime} h,
$$


where $\rho$ is a reference density of the air and $g^{\prime}$ is the reduced gravity defined in terms of the temperature difference between the interior and exterior, $\Delta T$, as

$$
g^{\prime}=\frac{\Delta T g}{T}
$$

for air (with $T$ measured in $\mathrm{K}$ ), and as

$$
g^{\prime}=\frac{\beta g \Delta T}{\rho}
$$

for water, where $\beta$ is an expansion coefficient, with units of $\mathrm{kg} \mathrm{m}^{-3} \mathrm{~K}^{-1}$ (Turner 1973). Finally, we denote the magnitude of the pressure difference across the lower downwind opening by $\Delta p_{i}$. Note the actual difference in pressure on the inner and outer surfaces of this opening changes sign depending on the flow regime.

For each flow regime (figure 2), the system is described by an overall mass balance and an energy balance, combined with the law for the volume flow through each of the three openings (equation (1)). This gives us five equations for the five unknown quantities $\Delta p_{i}, \Delta T, Q_{1}, Q_{2}$ and $Q_{3}$, in terms of the wind forcing $\Delta p_{w}$, the internal heat load $H$, and the fluid properties and building geometry.

We first consider the wind-driven regime (figure $2 c$ ). Since the changes in density associated with the temperature changes are small, we can write the mass balance in the approximate form (Batchelor 1967)

$$
Q_{1}=Q_{2}+Q_{3}
$$

Combining equations (1) and (5) and noting the pressure differences across each opening, we find:

$$
A_{1} \sqrt{\Delta p_{w}-\Delta p_{b}-\Delta p_{i}}=A_{2} \sqrt{\Delta p_{b}+\Delta p_{i}}+A_{1} \sqrt{\Delta p_{i}} .
$$

It is convenient to use dimensionless variables. We define $\alpha=A_{2} / A_{1}$, the ratio of the area of the upper upwind opening to the area of the downwind upper opening. We scale pressure relative to the wind forcing $\Delta p_{w}$, setting

$$
p=\frac{\Delta p_{i}}{\Delta p_{w}}
$$

and

$$
\theta=\frac{\Delta p_{b}}{\Delta p_{w}}
$$

We can then rearrange equation (6) to express $p$ in terms of $\theta$ and $\alpha$ :

$$
p=\frac{2-\alpha^{2} \theta-\alpha^{4} \theta+\alpha^{2}-2 \theta-2 \sqrt{\alpha^{2}\left(1-\alpha^{2} \theta-\theta^{2}\right)}}{4+\alpha^{4}} .
$$

We then use an energy balance to equate heat lost (as warm air leaves the building) to heat input (from the building heat sources).

$$
H=\rho C_{p} Q_{1} \Delta T,
$$

where $H$ is heat flux, $\rho$ is fluid density, $C_{p}$ is specific heat capacity, and $\Delta T$ is the temperature difference between the interior and exterior fluids. Note that $Q_{1}$ is equal to the volume flux out of the building (see equation (5)).

By combining equations (1), (9) and (10), and considering the pressure difference driving flow into the building through the upper upwind opening, we obtain the 
following dimensionless relationship between the internal-external temperature difference $\theta$ and the heat input to the room $q$ :

$$
q=\theta \sqrt{1-p-\theta},
$$

where $q=H \beta g h /\left(\sqrt{\rho} C_{p} c^{*} A_{1} \Delta p_{w}^{3 / 2}\right)$ is a dimensionless heat flux which compares actual heat flux with heat flux required to generate a ventilation flow equal to that provided by the wind, and $p(\theta)$ is given by equation (9).

The variation of $\theta$ as a function of $q$ is found by solving the coupled nonlinear algebraic equations (9) and (11). The solutions will be described in $\S 3$. First, we present equivalent relations to describe the temperature and flow in the intermediate (figure $2 b$ ) and buoyancy-driven (figure $2 c$ ) regimes.

For the intermediate regime:

$$
q=\theta \sqrt{\theta-p}
$$

where

$$
p=\frac{\alpha^{3} \theta-\alpha+3 \alpha \theta-2 \sqrt{\alpha^{2} \theta+2 \theta-1-\theta^{2}}}{\alpha\left(4+\alpha^{2}\right)} .
$$

For the buoyancy-driven regime:

where

$$
q=\theta \sqrt{p},
$$

$$
p=\frac{\alpha^{2}-2+2 \theta-\alpha^{2} \theta+\alpha^{4} \theta+2 \sqrt{\alpha^{2}\left(\theta^{2}-\alpha^{2} \theta+1\right)}}{4+\alpha^{4}} .
$$

We now use these relations to describe the possible differences between the internal and external temperature for any given heat load.

\section{Predictions}

In figure 4 , we show how the dimensionless temperature $\theta$ varies as the dimensionless heat input $q$ increases, for various values of the ratio of the upper and lower vent areas $\alpha$. The solid lines in figure 4 represent wind-driven regimes in which $\mathrm{d} \theta / \mathrm{d} q>0$. The long-dashed lines represent wind-driven regimes in which $\mathrm{d} \theta / \mathrm{d} q<0$. The short-dashed lines represent the intermediate regime (figure $2 b$ ), and the dotted lines represent the buoyancy-driven regime (figure $2 a$ ). The transitions between these regimes are plotted for four different opening ratios $\alpha ; 0,0.4,0.7$ and 1 , reading from left to right on the graph.

Figure 5 shows temperature $\theta$ as a function of heat input $q$ for the two-opening case, i.e. $\alpha=0$. The thick dotted line represents the buoyancy-driven case, and the thick dashed and thick solid lines represent the wind-driven case (see figure 1). If $q$ is sufficiently small $\left(0<q<q_{m}\right)$, then the figure shows that three solutions exist, labelled $(a),(b)$ and $(c)$. To investigate the stability of each solution, we consider the transient behaviour of the system for temperature close to the steady-state solutions. The overall heat balance of the system leads to heating or cooling of the interior fluid depending on whether the heat supply $H$ exceeds the heat which is convected from the space by the ventilation flow. We use this heat balance along with a mass balance for the building and equations (1) and (2) to show that:

$$
\rho C_{p} V \Delta \dot{T}=H-\rho C_{p} c^{*} A \Delta T \sqrt{\frac{\Delta p_{w}-\beta g h \Delta T}{2 \rho}}
$$




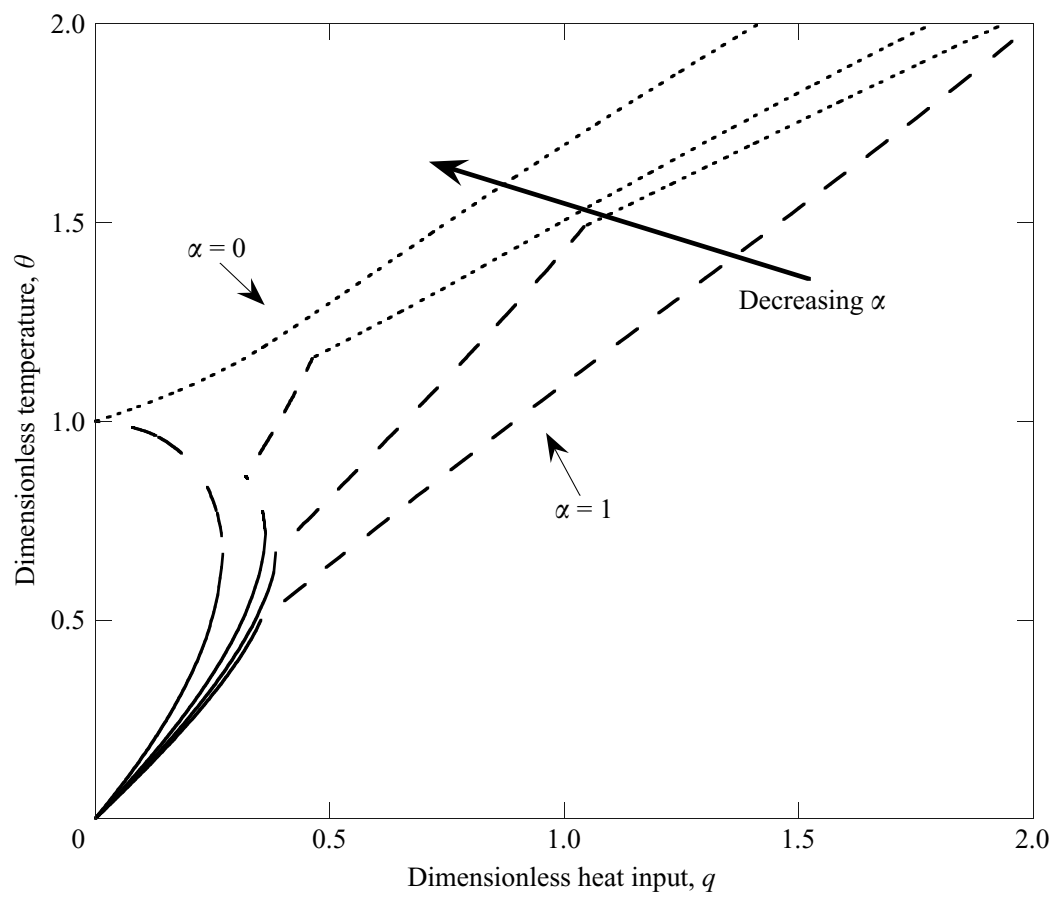

FiguRE 4. Temperature as a function of heat input for various area ratios $\alpha$. The four curves correspond to the parameter values, from left to right, $\alpha=0, \alpha=0.4, \alpha=0.7$ and $\alpha=1$. The solid lines represent the stable wind-driven regime (figure $2 c$ ), the long-dashed lines represent the unstable wind-driven regime (figure $2 c$ ), the short-dashed lines represent the intermediate regime (figure $2 b$ ), and the dotted lines represent the buoyancy-driven regime (figure $2 a$ ). At low values of $\alpha$, multiple steady states exist for certain values of $q$.

for the wind-driven regime, and

$$
\rho C_{p} V \Delta \dot{T}=H-\rho C_{p} c^{*} A \Delta T \sqrt{\frac{\beta g h \Delta T-\Delta p_{w}}{2 \rho}}
$$

for the buoyancy-driven regime. In dimensionless form (see equations (7) and (8)) we can write:

$$
C_{1} \dot{\theta}=\sqrt{2} q-\theta \sqrt{1-\theta}
$$

for the wind-driven regime, and

$$
C_{1} \dot{\theta}=\sqrt{2} q-\theta \sqrt{\theta-1}
$$

for the buoyancy-driven regime, where $C_{1}=\sqrt{2 \rho} V /\left(\sqrt{\Delta p_{w}} c^{*} A\right)$ has units of time. This transient behaviour is plotted in figure 6, which shows a plot of $\mathrm{d} \theta / \mathrm{d} t$ as a function of $\theta$ for a building with two openings, i.e. $\alpha=0$, for a typical dimensionless heat input $q=0.25$. The three possible steady-state solutions, (marked $(a),(b)$ and $(c)$ on the plot, and corresponding to points $(a),(b)$ and $(c)$ on figure 5) occur when $\mathrm{d} \theta / \mathrm{d} t=0$. We see from the graph that in the wind-driven solution on the left-hand side, $(a)$, and in the buoyancy-driven solution, $(c)$, any deviation from the steady state will decay, but in the wind-driven solution on the right-hand side, $(b)$, any deviation will grow and the system evolves to another regime. We therefore conclude that the second part of the wind-driven regime, where $\mathrm{d} \theta / \mathrm{d} q<0$, shown on figure 4 as a long-dashed line, 


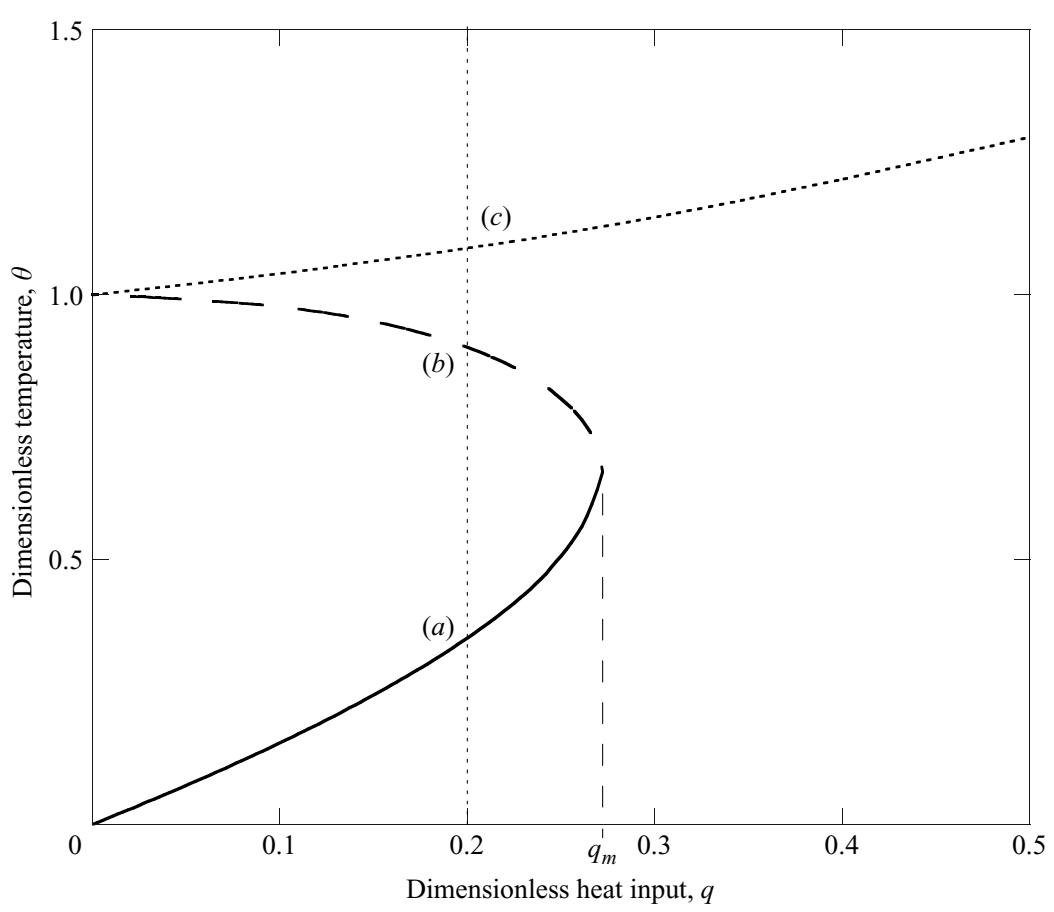

Figure 5. Temperature as a function of heat input for area ratio $\alpha=0$. The dotted line represents the buoyancy-driven regime while the dashed and solid lines represent the wind-driven regime (see figure 1). $q_{m}$ is the maximum temperature at which multiple steady states are possible. The thin dotted line represents a typical heat load for which three steady states can occur; these three states are labelled $(a),(b)$ and $(c)$.

is unstable in the case with two openings, and by continuity, in the case with three openings.

Figure 4 reveals that at low values of $\alpha$, when the upper downwind opening area is small, there is a range of heat fluxes for which we observe multiple steady states. As $\alpha$ increases, the range of dimensionless heat fluxes for which the unstable mode (the long-dashed line) develops becomes smaller; instead the range of heat fluxes for which the intermediate mode (the short-dashed line) develops becomes larger. Eventually, at a critical value of $\alpha, \alpha=\alpha_{c}$, say, multiple steady states cease to occur and for $\alpha>\alpha_{c}$ only one temperature is possible for any given heat input.

This information is represented in figure 7 , in which the possible flow regimes are shown as a function of the dimensionless area $\alpha$ and the dimensionless heat input $q$. In figure 8 , we illustrate the same transitions in flow regime on a graph of dimensionless temperature $\theta$ as a function of dimensionless heat input $q$, to complement the regime diagram of figure 7. The marked boundaries in figure 8 correspond to those of figure 7.

Figure 8 shows temperature as a function of heat input, for various values of window area ratio $\alpha(\alpha=0,0.4,0.7,1$, as in figure 4$)$. In figure 8 , the triangles represent the maximum heat input for which the wind-driven regime can occur. The squares represent the transition from wind-driven to intermediate, and hence the minimum heat input for which the intermediate regime occurs. The circles represent the transition from intermediate to buoyancy-driven regimes. The lines in figure 7 


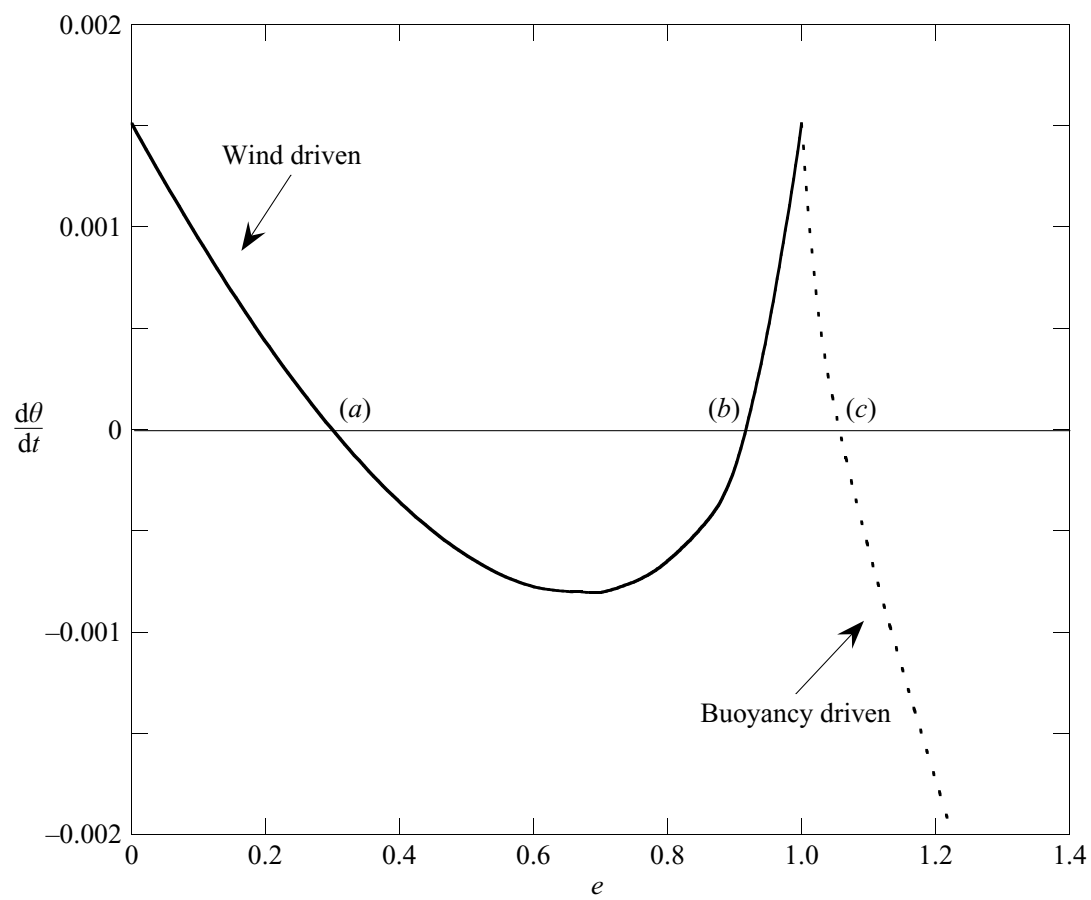

FIGURE 6. $\mathrm{d} \theta / \mathrm{d} t$ as a function of $\theta$ for a building with two openings, showing the stability of the possible regimes. The three possible steady-state solutions $(a),(b)$ and $(c)$ correspond to the wind-driven solutions (points $(a)$ and $(b)$ ) and the buoyancy-driven solution (point $(c)$ ) as shown in figure 5. Near to solution $(a), \mathrm{d}(\mathrm{d} \theta / \mathrm{d} q) / \mathrm{d} \theta<0$, and so any small deviation from the equilibrium $\theta=\theta_{e}$ is stable. Similarly, it may be seen that the buoyancy-driven solution, $(c)$, is stable, but the second wind-driven solution, $(b)$, is unstable.

correspond to the loci of the triangles, squares and circles in figure 8 , and this provides further insight into the conditions under which multiple steady states can exist.

The line labelled W (on figures 7 and 8) represents the maximum heat input for which a wind-driven regime can exist; line I represents the minimum heat input for which an intermediate regime can occur; and line IB represents conditions under which there is a transition from the intermediate to the buoyancy-driven flow regimes.

This figure illustrates that as $\alpha$ increases, the range of values of heat input $q$ for which multiple steady states exist becomes smaller. Eventually, when $\alpha=\alpha_{c}$ there is a unique solution for all values of $q$.

The minimum area ratio at which multiple steady states cease to exist corresponds to the situation in which the point of transition from the wind to the intermediate mode corresponds to the maximum heat load for which a wind-driven regime arises.

From equation (11), the maximum value of $q$ for which a wind-driven regime exists occurs when

$$
\frac{\mathrm{d}}{\mathrm{d} \theta}(\theta \sqrt{1-p-\theta})=0
$$

where $p$ is given by equation (9).

At the transition between wind-driven and intermediate regimes, there is no flow through the lower opening, and hence

$$
\Delta p_{i}=0 .
$$




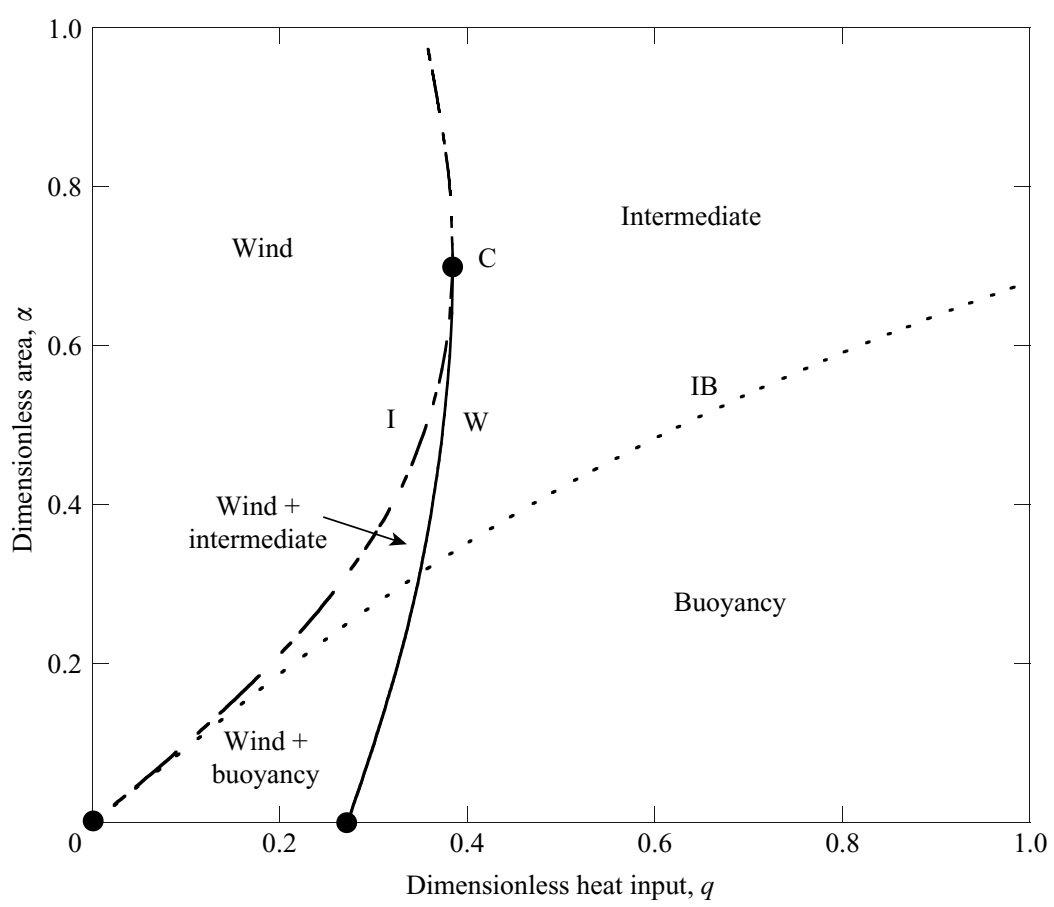

FIGURE 7. Regime diagram, shown on a plot of ratio of openings $\alpha$ as a function of dimensionless heat input $q$. The solid line, labelled $\mathrm{W}$, represents the maximum heat load for which a wind-driven solution is possible. The dashed line, labelled I, represents the minimum heat load for which an intermediate solution is possible. The dotted line, labelled IB, represents the transition from the intermediate regime to the buoyancy-driven regime. In three regions of the diagram (labelled wind, intermediate and buoyancy, respectively), only one flow regime is possible. In two regimes (labelled wind + intermediate and wind + buoyancy, respectively), multiple steady states exist, as labelled.

Combining equations (21) and (6) gives a relationship between $\theta$ and $\alpha$ at the transition point:

$$
\theta=\frac{1}{1+\alpha^{2}}
$$

Combining equations (9), (20) and (22) and solving for $\alpha$, we find the critical area ratio,

$$
\alpha_{c}=\frac{1}{\sqrt{2}} .
$$

We deduce that if the area of the upper downwind opening is at least $1 / \sqrt{2}$ times that of the other two window openings, multiple steady states will not occur.

There is also a critical heat input, say $q_{c}$, above which multiple steady states cannot occur. To find this, we define the critical point $\mathrm{C}$, at which the transition from the wind-driven regime to the intermediate regime changes from going through multiple steady states to switching directly (marked $\mathrm{C}$ on figures 7 and 8).

Returning to equation (22), we can find a value for the dimensionless temperature at $\mathrm{C}$ :

$$
\theta_{c}=\frac{2}{3}
$$




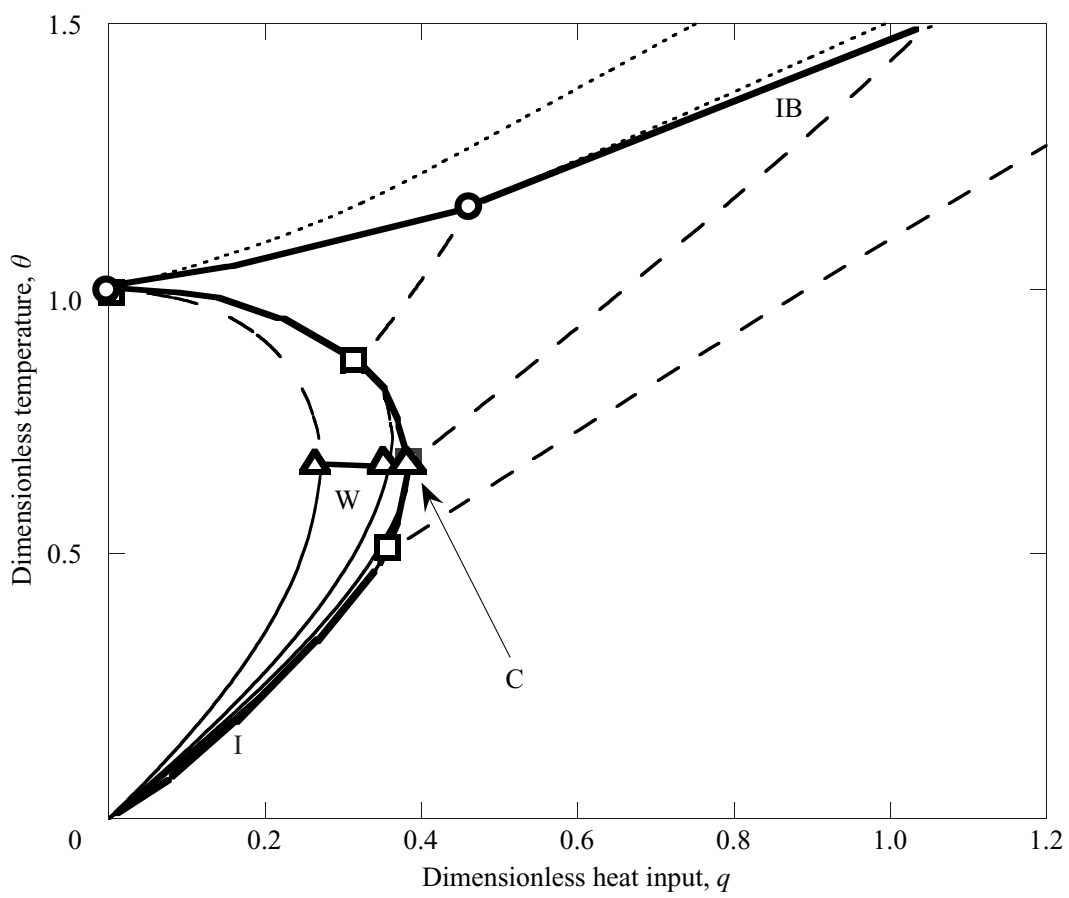

FiguRE 8. Graph to illustrate the regime boundaries shown in figure 7 , in the $(\theta, q)$-plane. The triangular markers represent the maximum of the wind-driven regime for each curve for a series of values of $\alpha$, and the line intersecting these corresponds to the solid line labelled $W$ on figure 7. The square markers represent the transitions from the wind-driven regime to the intermediate regime, and hence the minimum possible temperature at which the intermediate regime can occur, for a series of values of $\alpha$. The line joining these squares corresponds to the line labelled I in figure 7. The circular markers represent the transition from the intermediate regime to the buoyancy-driven regime for a series of values of $\alpha$, and the line joining these markers corresponds to line IB in figure 7. In this figure, we have also included a series of thin lines to illustrate the solutions $\theta(q)$ for a series of values of $\alpha$ as shown in figure 4 .

and from equation (11) we find the dimensionless heat flux,

$$
q_{c}=\frac{2 \sqrt{3}}{9},
$$

at the critical point. For heat loads in excess of $q_{c}$, there exists a unique steady solution at all times.

\section{Experiments}

The theory is tested empirically using an analogue building model contained in water in a recirculating flume (figure 9). The analogue experimental system consists of a small Perspex tank, the model building, of dimensions $17.65 \times 17.65 \times 17.65 \mathrm{~cm}^{3}$, with seven circular openings (radius $0.75 \mathrm{~cm}$ ) at the top and bottom of each face. During the experiments, rubber bungs were used to seal different sets of openings. As an analogue experimental model of the two-opening flow regime, $\alpha=0$ (figure 1), the tank was set up with a row of openings at a high level on the upwind side and a row of openings at a low level on the downwind side. To model the case $\alpha=1$ (figure 2), a further row of openings was used at a high level on the downwind side. The model 


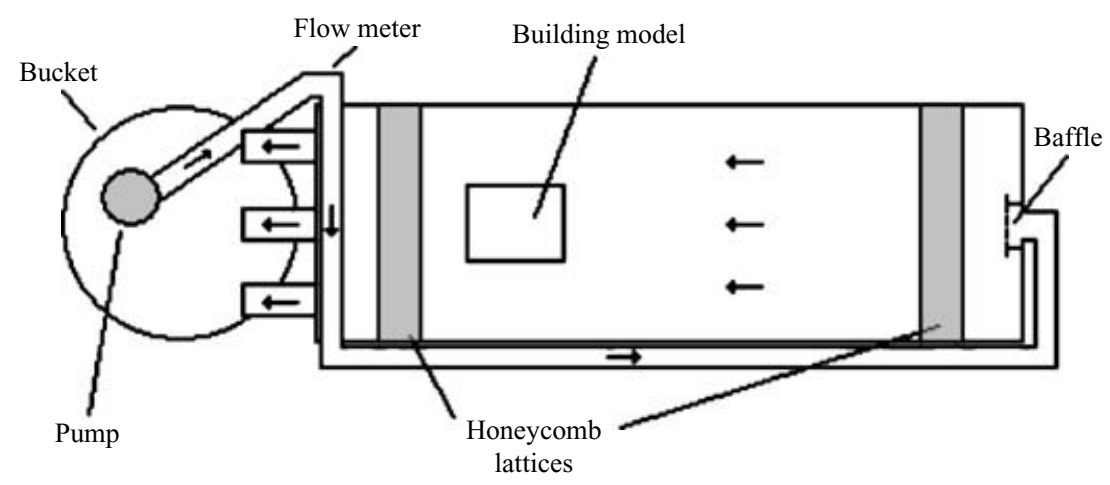

FIGURE 9. Experimental layout: the building model is placed in a flume tank, and water is pumped along the flume tank as an analogue to wind. Honeycomb lattices ensure uniform flow.

building is placed in a flume of dimensions $2 \times 0.5 \times 0.5 \mathrm{~m}^{3}$, which pumps water across the building and acts as a simple analogue of the wind, in that it produces a pressure decrease from the upflow face to the downflow face of the building. The flume contains honeycomb lattices to ensure a uniform flow as confirmed by observing the transport of dye streaks. Typical flow speed in the flume was around $1 \mathrm{~cm} \mathrm{~s}^{-1}$. The building is raised from the floor of the tank. The building model has a heating plate on its floor which represents a distributed heat source. The heating plate is made with a series of high-resistance metal coils attached to a power supply with variable power output (Gladstone \& Woods 2001).

Temperatures were measured using four type-K thermocouples vertically distributed within the building interior, and three thermocouples distributed in the exterior flume, to measure external temperature and ensure uniform conditions were maintained. Temperature measurements were made every $5 \mathrm{~s}$, using Picolog software via a TC- 08 cold junction, until the system reached steady state. The external temperature was around $35^{\circ} \mathrm{C}$, since the pump supplied a significant amount of heat to the system. It was ensured that the exterior fluid was in equilibrium before any data points were taken. Temperature measurements were made over $100 \mathrm{~s}$ and averaged to minimize the effects of fluctuations in the flume. Temperature differences within the building were found to be small $\left( \pm 0.1^{\circ} \mathrm{C}\right)$ compared to internal-external temperature differences (up to $7^{\circ} \mathrm{C}$ ), and did not increase with the heating load, suggesting that the interior was, to good approximation, well mixed. As well as quantitative temperature measurements, qualitative information about flow patterns was obtained from dye and shadowgraph techniques (for example, dye can be injected on either side of an opening to investigate flow direction through that opening). The fitting parameter $c^{*}$ was measured for the building model using a calibration experiment with no wind, where $c^{*}$ is the only parameter not independently measured, and found to be $c^{*}=0.95$. The pressure difference across the building associated with a given flow in the flume was measured using a series of calibration experiments in which the flow-induced pressure acted in parallel with the buoyancy (cf. Hunt \& Linden 2004). The wind pressure was found to be $p_{w}=1.9 \mathrm{~Pa}$ at the pump's maximum flow rate. Also, by performing calibration experiments testing the upper and lower openings independently, it was found that the pressure variation across each face was only a small percentage of the pressure difference between the upwind and downwind faces. All other parameters 
(a)

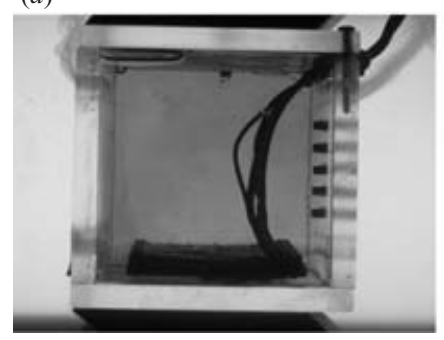

(b)

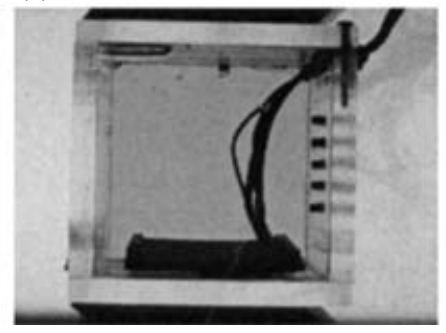

(c)

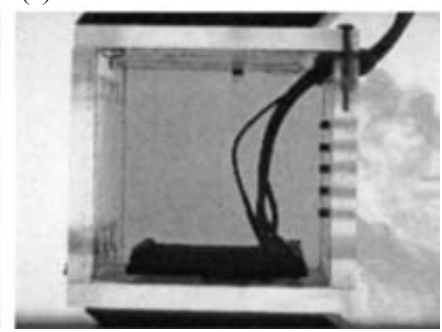

FIGURE 10. Experimentally observed regimes, for a building with three openings $(\alpha=1)$. (a) The buoyancy-driven regime, with outflow from both upper vents; $(b)$ the intermediate regime, with outflow from the upper downwind vent, and $(c)$ the wind-driven regime, with outflow through both downwind vents.

(i.e. building geometry) are independently measured. Other experimental values used were $\beta=0.36 \mathrm{~kg} \mathrm{~m}^{-3} \mathrm{~K}^{-1}, \rho=1000 \mathrm{~kg} \mathrm{~m}^{-3}, C_{p}=4184 \mathrm{~J} \mathrm{~kg}^{-1} \mathrm{~K}^{-1}$, and $g=9.81 \mathrm{~m} \mathrm{~s}^{-2}$.

First, experiments were performed in which there was one row of openings at a high level on the upwind side, and one row of openings at a low level on the downwind side, in order to test the prediction that multiple steady states can occur with $\alpha=0$ (figure 1). Experimental results with $\alpha=0$ are presented in dimensional form in figure 11. The solid line represents the stable wind-driven theory, while the circles represent data points corresponding to the wind-driven regime. These data points were obtained by starting with zero heat input and gradually increasing the heat input between measurements. Eventually, a transition to the buoyancy-driven regime occurred. The dashed line shows the unstable wind-driven solution. The dotted line shows the buoyancy-driven solution, and the squares show data points obtained in the buoyancy-driven regime. These were obtained by starting with the maximum achievable heat load and gradually decreasing until the mode changed. For all data points, the error bars represent the tolerance of the thermocouples used to measure temperature $\left( \pm 0.1^{\circ} \mathrm{C}\right)$ and the vertical stratification in the $\operatorname{tank}\left(<0.1^{\circ} \mathrm{C}\right)$.

Next, a third row of openings was included on the upper part of the downwind face of the tank to test the predictions of the model developed in $\S 2$ and presented in $\S 3$ (figure 2). Theoretical and experimental results for the case with three equal windows (i.e. $\alpha=1$ ) are presented in dimensional form in figure 12. Initially, the heat input was set at zero. Each successive data point was then found by increasing the heat input and waiting for the system to reach steady state. Similar results were also obtained by starting with a high heat input and gradually reducing it for each data point. Shadowgraph and dye techniques were used to observe the flow regimes. Of the data points on the graph, circles represent the wind-driven regime, squares the intermediate regime, and triangles the buoyancy-driven regime. The experimental data are shown alongside the theory: the solid line represents the wind-driven theory, the dashed line the intermediate, and the dotted line the buoyancy-driven regime (as in figure 2). Photographs of the experiments, showing the buoyancy-driven, intermediate, and wind-driven regimes, are shown in figure 10.

In both cases, experimental results are in good accord with the theory. The twoopening results show the hysteresis associated with multiple steady states and an unpopulated area associated with the unstable regime. There is a slight systematic discrepancy between the theory and experiment in the buoyancy-driven regime for the two-opening case (figure 11), as the difference between the unstable wind-driven regime and the stable buoyancy-driven regime becomes small. 


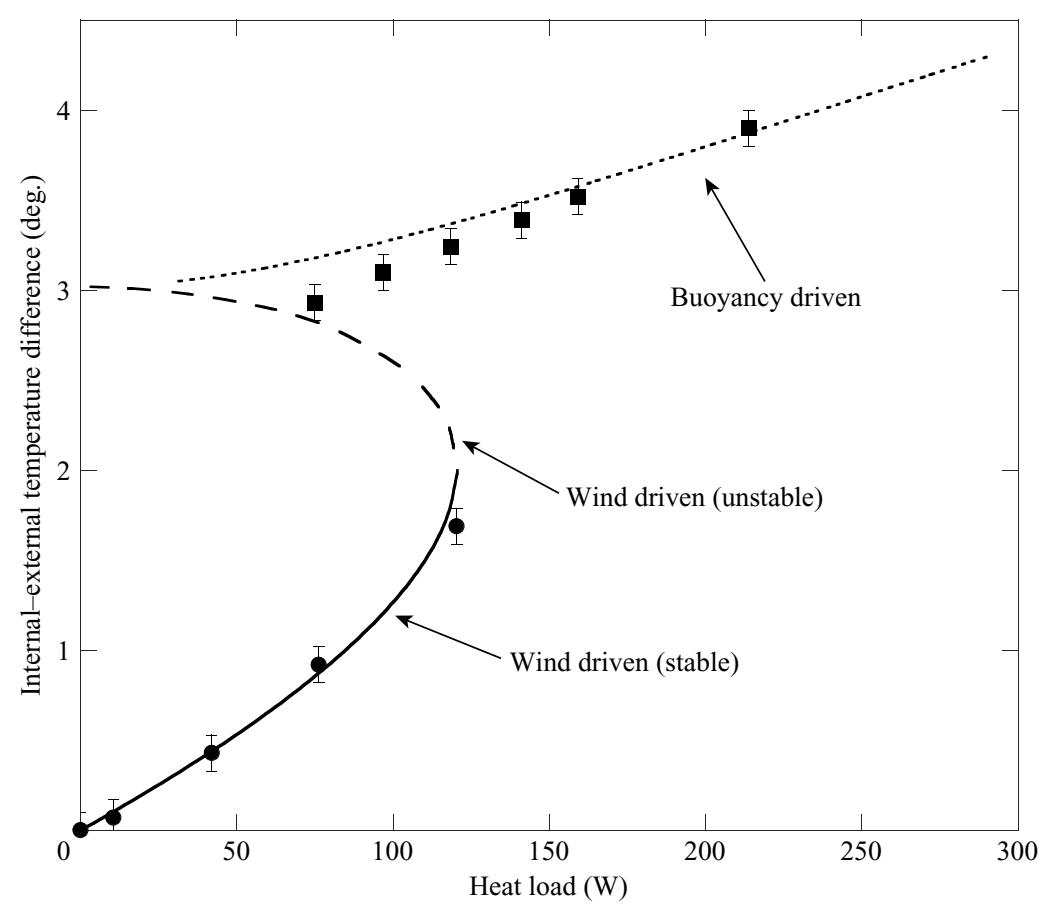

FIGURE 11. Graph of internal-external temperature difference $\Delta T$ (in ${ }^{\circ} \mathrm{C}$ ) as a function of heat load $Q$ (in $\mathrm{W}$ ), showing theory and experiment, for a building model with two openings of equal area (see figure 1). The solid line represents the theoretical stable wind-driven solution, the dashed line the unstable wind-driven solution, and the dotted line the buoyancy-driven solution. The circular markers show experimental data points in the wind-driven regime, and the square markers represent experimental data points in the wind-driven regime. The error bars represent the tolerance of the thermocouples used to measure temperature $\left( \pm 0.1^{\circ} \mathrm{C}\right)$ and the vertical stratification in the tank $\left(<0.1^{\circ} \mathrm{C}\right)$.

The experimental system, in fact, has seven openings on the upwind face and fourteen openings on the downwind face, with seven located at the upper elevation and seven at the lower elevation. Near the point of transition between the wind and buoyancy dominated flow, the pressure change across one set of these openings becomes small. As a result, any small horizontal pressure fluctuations within the tank, which can lead to a gradient in the pressure difference across the openings at a given height, may become dominant. Indeed, this may then lead to inflow through some openings and outflow through other openings at the same elevation. Such threedimensional effects are not included in the present model, but may account for the difference between the model prediction and the data. This interpretation is supported by the observation that near the point of transition in flow regime, the flow reverses through each of the openings at a given elevation in sequence, rather than occurring en masse.

\section{Effect of varying height of new opening}

The analysis presented so far has assumed the height of the upper downwind opening is the same as that of the upper upwind opening. We now show how the analysis may be generalized to account for situations in which the new opening is at a different height. We define the height of the upper upwind opening as $h_{1}$, the 


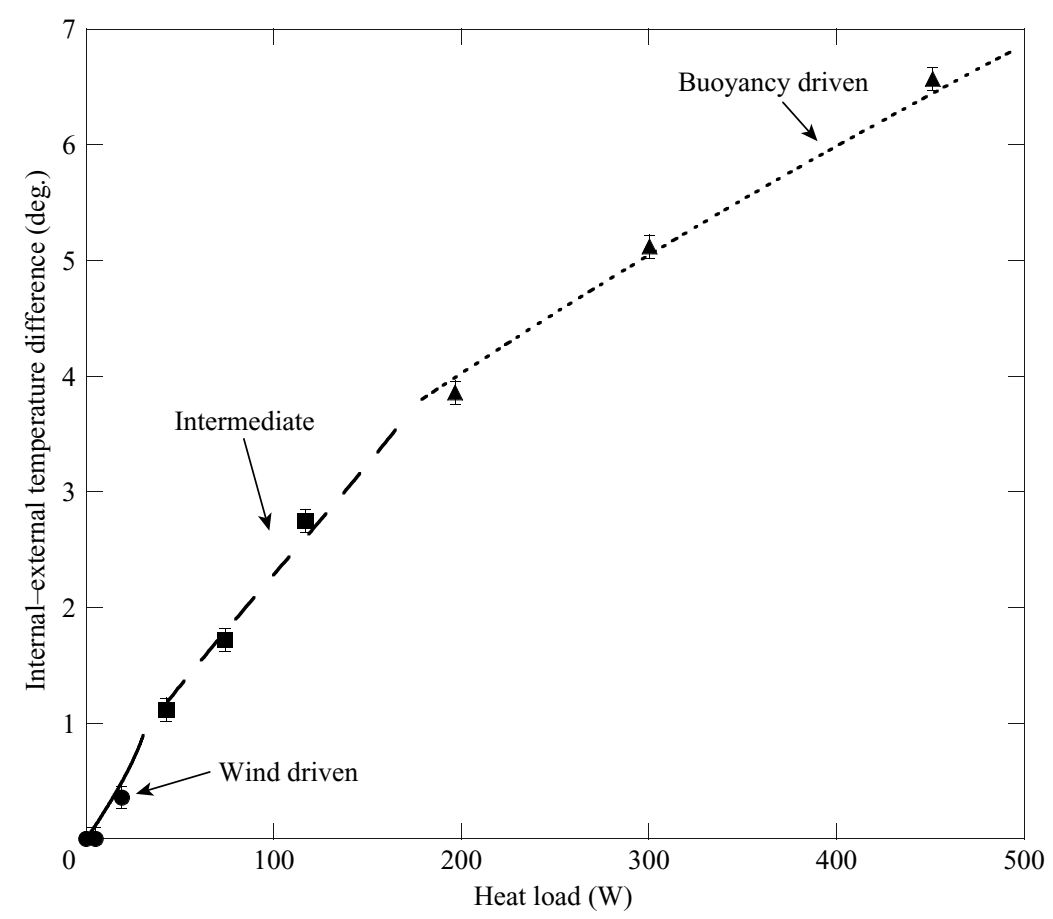

FIGURE 12. Graph of internal-external temperature difference $\Delta T$ (in ${ }^{\circ} \mathrm{C}$ ) as a function of heat load $Q$ (in $W$ ), showing theory and experiment, for a building model with three openings of equal area (see figure 2). The solid line represents the theoretical wind-driven solution, the dashed line the intermediate solution, and the dotted line the buoyancy-driven solution. The circular markers correspond to experimental data points in the wind-driven regime, the square markers to experimental data points in the intermediate regime, and the triangular markers to experimental data points in the buoyancy-driven regime. The error bars represent the tolerance of the thermocouples used to measure temperature $\left( \pm 0.1^{\circ} \mathrm{C}\right)$ and the vertical stratification in the tank $\left(<0.1^{\circ} \mathrm{C}\right)$.

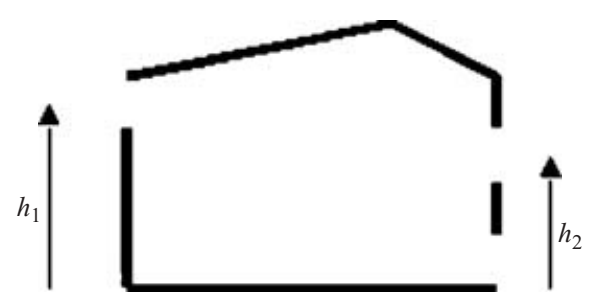

FIGURE 13. The new building model with the upper downwind opening at a variable height: the height ratio $\omega=h_{2} / h_{1}$.

height of the upper downwind opening as $h_{2}$, and the ratio $h_{2} / h_{1}=\omega$ (see figure 13). Where the upper downwind opening is at a position $h_{2}<h_{1}$, the three flow regimes described earlier are possible, but a new fourth buoyancy-driven regime can also develop in which there is inflow through both of the downwind openings and outflow from the upwind opening (figure 14). In the case of zero wind, the flow through the intermediate-elevation vent can be either in- or outflow depending on its height in relation to the neutral pressure level in the room (Fitzgerald \& Woods 2004). This 
(a) (i)

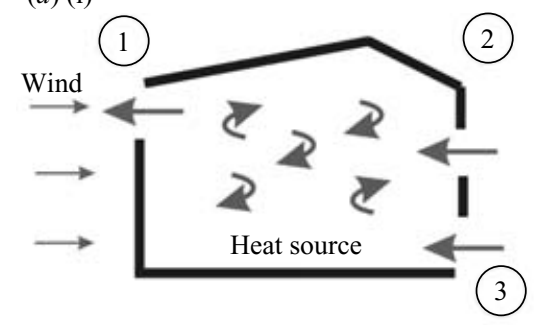

(b)

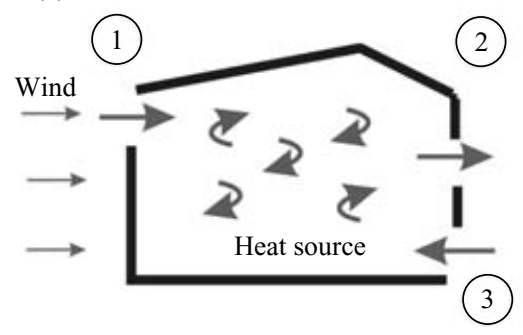

(ii)

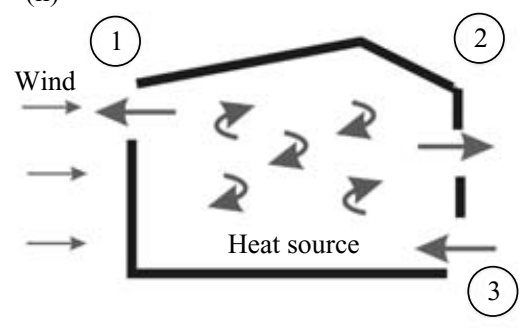

(c)

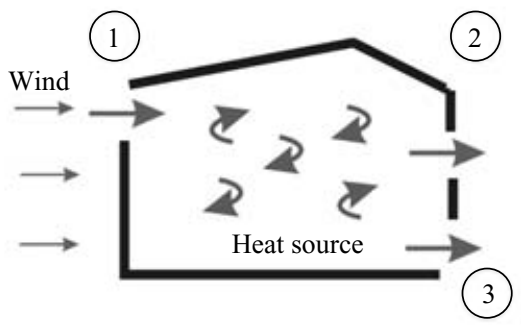

FIGURE 14. (a) Buoyancy driven; (b) intermediate; $(c)$ wind driven. Possible flow regimes: when the height of the upper upwind opening is varied, a new buoyancy-driven regime (shown here as $(a)(\mathrm{i}))$ can exist.

neutral pressure level is given for the case with no wind by

$$
z_{n}=\frac{A_{u}^{2}}{A_{l}^{2}+A_{u}^{2}} h_{b}=\Gamma h_{b},
$$

where $h_{b}$ is the vertical distance between the top and bottom openings, $A_{u}$ is the opening area of the top opening, and $A_{l}$ is the area of the bottom opening. If $\omega>\Gamma$, the flow through the upper downwind opening will be outflow, whereas if $\omega<\Gamma$, the flow will be inflow. If a wind pressure gradient develops across the building, then the neutral height will move to some new level, say $\Gamma_{w} h_{b}$. For $\omega>\Gamma_{w}$, as the heat load increases, the flow will evolve in a similar fashion to that shown in figure 2, from a wind-driven regime through an intermediate regime to a buoyancy dominated regime. If $\omega<\Gamma_{w}$, then, as the heat load increases, the flow will go through winddriven, intermediate and buoyancy-driven regimes as before, but now, with increasing heat load, a fourth regime becomes possible, in which flow is inwards through the downwind openings and outwards through the upwind opening (see figure $14 a(\mathrm{i})$ ).

To further investigate the effects of varying $\omega$, we use equation (2), and note that $\Delta p_{b}$ is proportional to height, $h$. Therefore, the ratio of two heights above the lower opening will equal the ratio of pressures due to density difference, i.e. the ratio of $\Delta p_{b}$ at those two different heights. We can therefore adapt equation (6) to give, for the wind-driven regime:

$$
\sqrt{1-\theta-p}=\alpha \sqrt{\omega \theta+p}+\sqrt{p},
$$

where $p(\theta)$ is calculated using a mass balance as in $\S 2$. Similarly, for the intermediate and buoyancy-driven regimes, we find

$$
\sqrt{1-\theta+p}+\sqrt{p}=\alpha \sqrt{\omega \theta-p}
$$




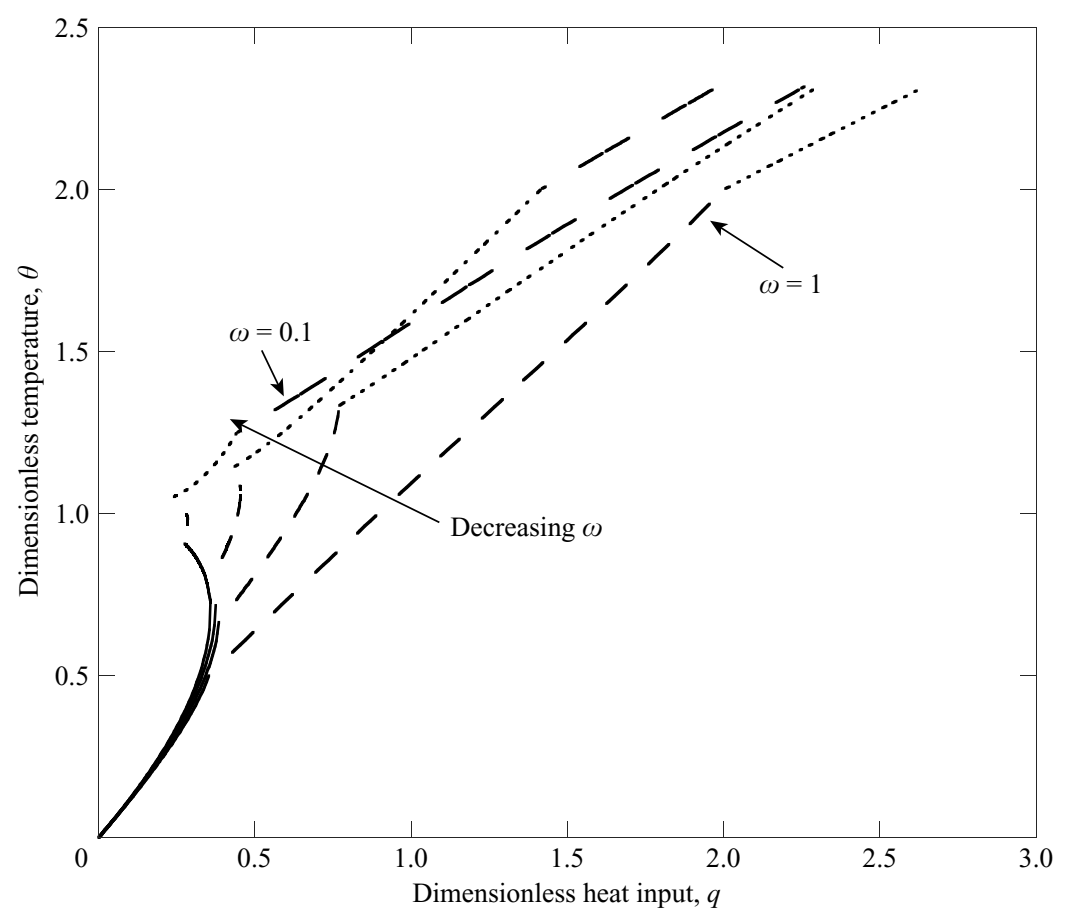

FIGURE 15. Temperature $\theta$ as a function of heat input $q$ for various height ratios $\omega$ (with area ratio $\alpha=1$ ). From right to left, the four curves represent $\omega=0.1, \omega=0.25, \omega=0.5$ and $\omega=1$. The long-dashed line shows the new buoyancy-driven regime (figure 14a(i)), the dotted line shows the original buoyancy-driven regime (figure $14 a(\mathrm{ii})$ ), and the short-dashed line shows the intermediate regime (figure 14b). The solid line represents the wind-driven regime (figure 14c) (here, both the stable and unstable modes are shown as a solid line).

and

respectively.

$$
\sqrt{1-\theta+p}+\alpha \sqrt{\omega \theta-p}=\sqrt{p}
$$

As before, we can then use equation (10) to find an expression for $q$ as a function of $\theta$ for each regime. Figure 15 shows the predictions of this model for varying $\omega$. For this figure, all openings are assumed to be of the same size, i.e. $\alpha=1$. We see that raising the height of the new window can eliminate multiple steady states in much the same way as an increase in area did. Again the figure shows a wind-driven regime (solid line), an intermediate regime (short-dashed line) and a buoyancy-driven regime (dotted line). Note the new fourth regime, with inflow through both downwind openings, which is shown by the long-dashed line. In this regime, a lower value of $\omega$ will lead to more ventilation, since the flow through the upper downwind opening is now inwards.

Figure 16 shows a regime diagram for the case when $\omega=0.4<\Gamma$, i.e. the upper downwind opening is below the neutral pressure level, and hence the flow through this opening can be inward as in figure 14(a)(i). Figure 16 shows, on a plot of $\alpha$ as a function of $\log q$, a long-dashed line (labelled I) which represents the minimum heat input for which the intermediate solution can exist, a solid line (labelled W) which represents the maximum heat input for which the wind-driven regime can exist, and a dotted line (labelled IB) which represents the transition from the intermediate 


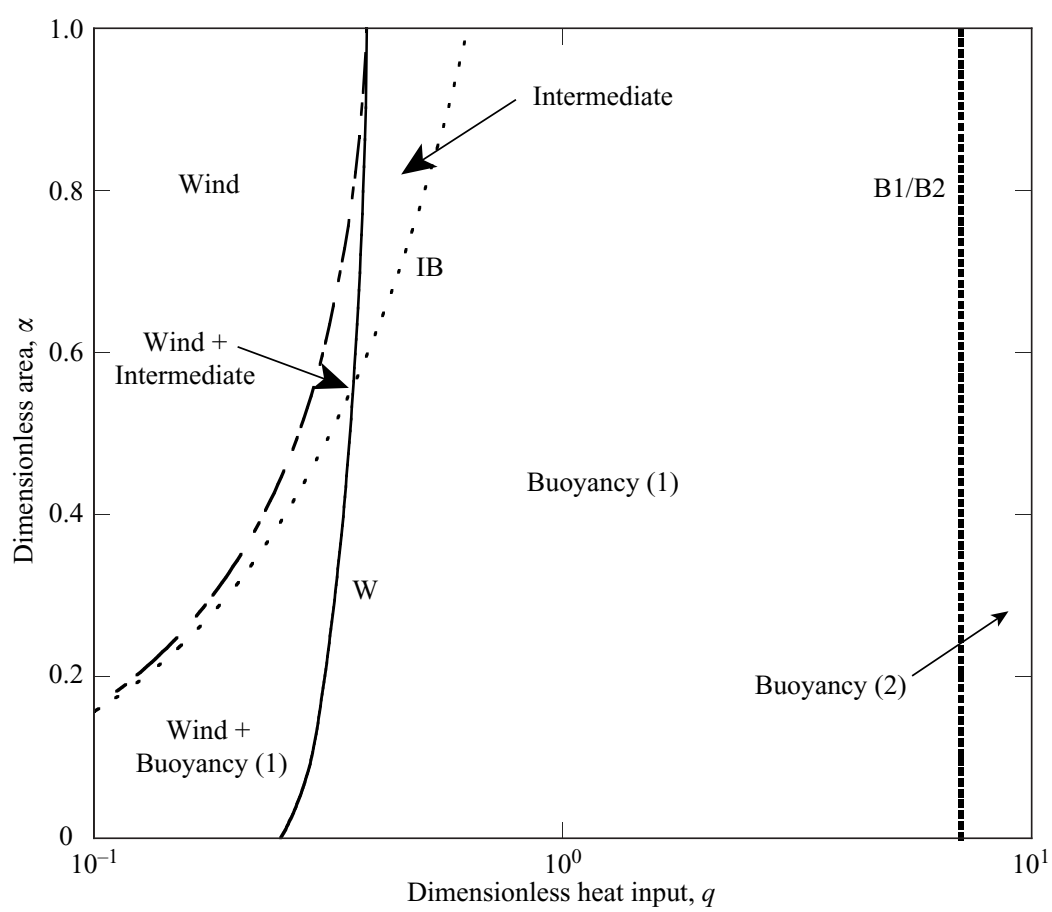

FIGURE 16. Regime diagram, shown on a plot of ratio of openings $\alpha$ as a function of the $\log$ of dimensionless heat input $q$, with height ratio $\omega=0.4$ (figure 7 shows a similar plot for $\omega=1$ ). The solid line, labelled $\mathrm{W}$, represents the maximum heat load for which a wind-driven solution is possible. The long-dashed line, labelled I, represents the minimum heat load for which an intermediate solution is possible. The dotted line, labelled IB, represents the transition from the intermediate regime to the buoyancy driven regime. The short-dashed line, labelled B1/B2, represents the transition between the two buoyancy regimes. In four regions of the diagram (labelled wind, intermediate, buoyancy (1) and buoyancy (2), respectively), only one flow regime is possible. In two regimes (labelled wind + intermediate and wind + buoyancy (1), respectively) multiple steady states exist, as labelled.

regime to the first buoyancy regime, as in figure 7. Also shown on the graph is the transition between the two buoyancy regimes (figure $14 a$ (ii) to $14 a(\mathrm{i})$ ), which occurs at a constant value of $q=7.07$ for $\omega=0.4$, independent of $\alpha$, and is labelled B1/B2.

To find the critical point $\alpha_{c}$ such that for $\alpha>\alpha_{c}$, there is a unique solution for all heat loads $q$, as before, we consider the wind-driven regime, combine equation (27) with a heat balance to give a relation between $q$ and $\theta$, and differentiate. At the critical point, then,

$$
\frac{\mathrm{d} q}{\mathrm{~d} \theta}=0 .
$$

This gives us one condition for the critical point $\mathrm{C}$. The other condition is found by considering the transition from the wind-driven regime to the intermediate regime: we now find that when the flow through the lower opening is zero,

$$
\theta=\frac{1}{1+\alpha^{2} \omega}
$$




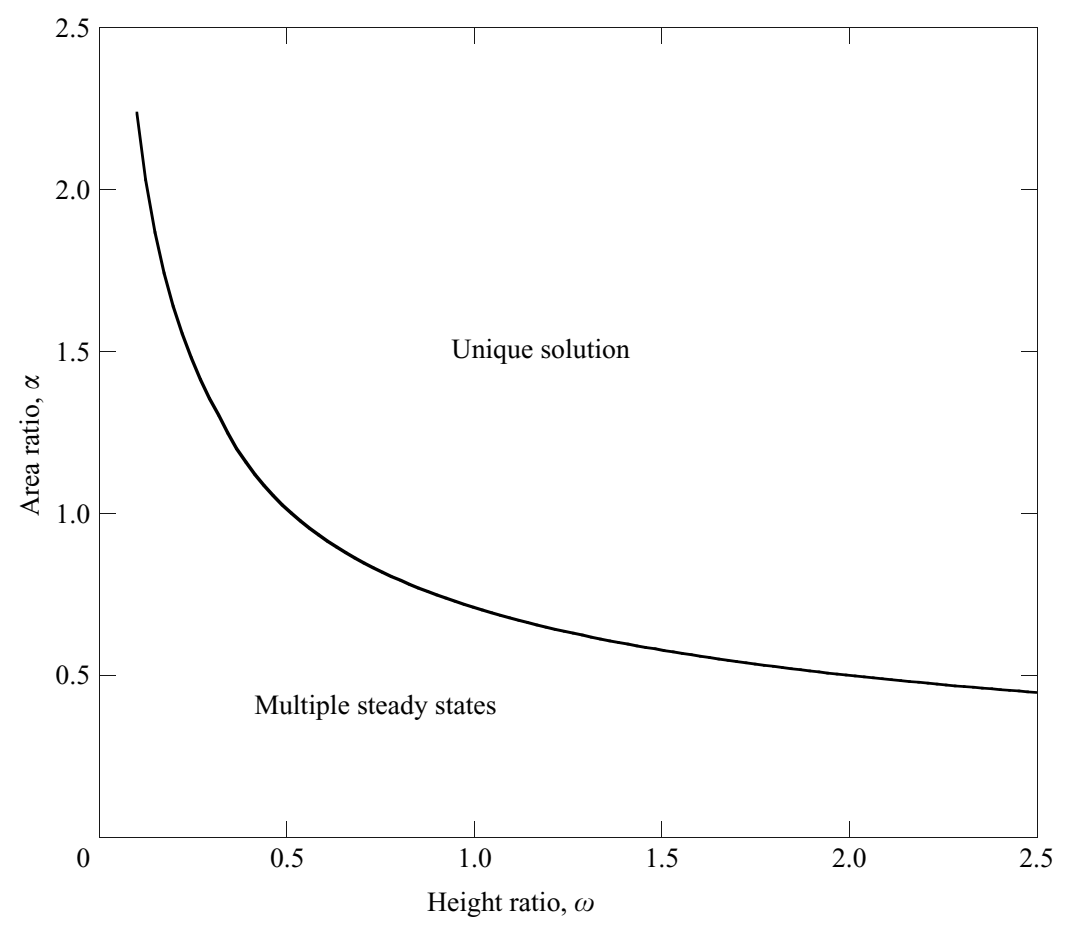

FIGURE 17. Plot of area ratio $\alpha$ as a function of height ratio $\omega$ summarizing regions of unique solutions and multiple steady states.

Combining these two conditions, we find the relationship between the height ratio $\omega$ and the area ratio $\alpha$ at the critical point:

$$
\alpha_{c}=\frac{1}{\sqrt{2 \omega_{c}}} .
$$

This relationship is plotted in figure 17, and summarizes our findings of the geometrical conditions under which there is always a unique flow regime, irrespective of the heat load.

\section{Applications and conclusions}

We now explore the predictions of the model in the context of a typical building. We consider a meeting room with an upwind window located $3 \mathrm{~m}$ above the floor, and a downwind opening at ground level, both of area $1 \mathrm{~m}^{2}$. We then examine the flow regime in the case where there is an additional opening 1,2 or $3 \mathrm{~m}$ above the floor on the downwind side of the building, and of size $0.8 \mathrm{~m}^{2}$. We assume that there is a pressure difference across the building of $0.8 \mathrm{~Pa}$ associated with the wind, perhaps corresponding to a wind speed of $2-3 \mathrm{~m} \mathrm{~s}^{-1}$. Figure 18 illustrates the predictions of the model for these three different configurations. The solid lines represent the wind-driven regime, the dashed lines the intermediate regime, and the dotted lines the buoyancy-driven regime. Note that when $\alpha=0.8$ and $\omega=0.33$ or $\omega=0.66$, figure 17 predicts multiple steady states, but when $\omega=1$ (the rightmost curve) we find a unique solution for all heat inputs.

Four possible heating loads are marked on the graph by thin dotted lines. The first (in the wind-driven regime) heat load has value $1.5 \mathrm{~kW}$, equivalent to 10 people 


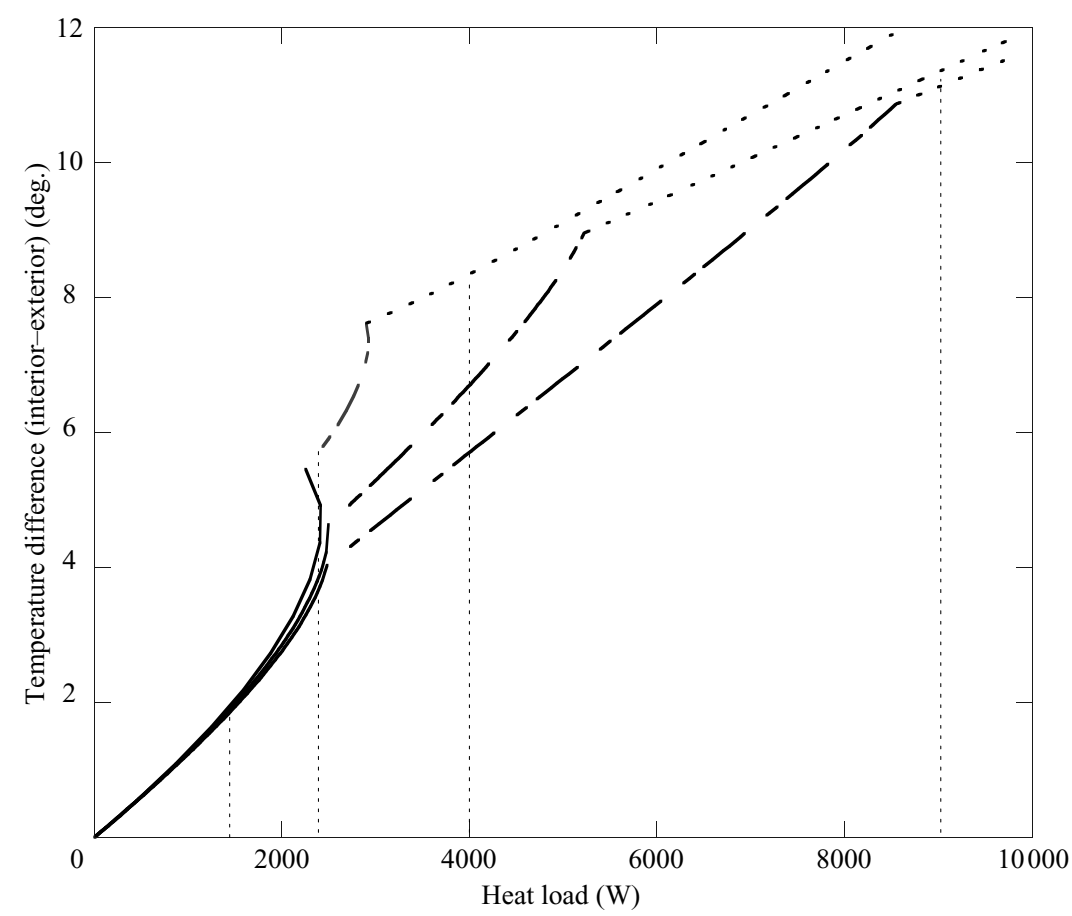

FIGURE 18. Dimensional plot of temperature difference (interior-exterior) in degrees Celsius as a function of heat load in watts. The building considered has an upwind opening at a height of $3 \mathrm{~m}$, and a downwind opening at $0 \mathrm{~m}$ (ground level) as well as an upper downwind opening at height $h_{d}$; plots are shown for (from left to right) $h_{d}=1 \mathrm{~m}, h_{d}=2 \mathrm{~m}$ and $h_{d}=3 \mathrm{~m}$, i.e. $\omega=0.33$, $\omega=0.66$ and $\omega=1$. The upper upwind and lower downwind windows have an area of $1 \mathrm{~m}^{2}$; the upper downwind opening has an area of $0.8 \mathrm{~m}^{2}$, i.e. $\alpha=0.8$. A typical wind pressure of $0.8 \mathrm{~Pa}$ acts on the face of the building. On each curve, the solid line represents the wind-driven regime, the dashed line the intermediate regime, and the dotted line the buoyancy-driven regime. The thin, vertical dotted lines represent possible operating heat loads: $1.5 \mathrm{~kW}, 2.4 \mathrm{~kW}, 4 \mathrm{~kW}$ and $9 \mathrm{~kW}$.

producing $150 \mathrm{~W}$ each, with no extra heating, which causes a temperature increase of around $2{ }^{\circ} \mathrm{C}$. This might be a typical summer load. The second heat load has value $2.4 \mathrm{~kW}$; at this heat load, multiple steady states can occur when $\omega=0.33$ or $\omega=0.66$. The third heat load marked is $4 \mathrm{~kW}$, which might represent a typical spring load, with some additional heating. At this heat load, with $\omega=0.66$ or $\omega=1$, the building will be in intermediate mode. Finally in the winter, a high heat load will generate a buoyancy-driven flow as shown for the case with an internal heat load of $9 \mathrm{~kW}$.

By designing the building such that multiple steady states cannot occur, each heat load can be mapped to a corresponding internal temperature (given the external temperature and wind), and hence temperature control in the building is simplified.

Naturally ventilated buildings are designed to provide a comfortable indoor environment while minimizing energy used in heating and cooling. Multiple steady states in naturally ventilated buildings complicate temperature control. It is well documented that multiple steady states can arise in buildings with two openings in the presence of wind.

This study has shown both theoretically and with new laboratory experiments that for a building with a distributed heat source in the presence of wind, the flow behaviour with three openings is fundamentally different. With three openings, it 
is possible for the flow direction through any opening to change while building ventilation continues through the other two openings. The transition between states may therefore be smooth (figure 2), and the multiple steady states associated with two openings (figure 1) may be eliminated. However, by continuity, if the area of the new opening is very small, the flow behaviour will be similar to the two-opening case, and exhibit multiple steady states; only as the area is increased to match the areas of the other two openings does the flow regime become uniquely defined. We have also shown that the critical size of the new opening, above which multiple steady states are no longer possible, increases as the elevation of the new opening above the lower downwind opening decreases.

Several fundamental issues still remain about these nonlinear natural ventilation flows, especially in the case with multiple states; amongst other studies, in the future we plan to extend our analysis by considering the transient evolution of the system to steady state, and the effects of time-dependent heat loads or external conditions.

\section{REFERENCES}

Batchelor, G. K. 1967 An Introduction to Fluid Dynamics. Cambridge University Press.

Department of Trade and Industry 2003 http://www.dti.gov.uk/renewables/policy/longertermoptions.pdf.

Fitzgerald, S. D. \& Woods, A. W. 2004 Natural ventilation of a room with vents at multiple levels. Build. Environ. 39, 505-521.

Gladstone, C. \& Woods, A. W. 2001 Buoyancy-driven natural ventilation of a room. J. Fluid Mech. 441, 293-314.

Hunt, G. R. \& Linden, P. F. 2000 Multiple steady airflows and hysteresis when wind opposes buoyancy. Air Infiltration Rev. 21, 1-3.

Hunt, G. R. \& Linden, P. F. 2004 Displacement and mixing ventilation driven by opposing wind and buoyancy. J. Fluid Mech. 527, 27-55.

Li, Y., Delsante, A., Chen, Z. \& Sandberg, M. 2000 Some examples of solution multiplicity in natural ventilation. Proc. 7th Intl Conf. on Air Distribution in Rooms, July 9-12 2000 Reading, UK, Vol. 1, pp. 289-294.

Linden, P. F. 1999 The fluid mechanics of natural ventilation. Annu. Rev. Fluid Mech. 31, 201-238.

Linden, P. F., Lane-SerfF, G. F. \& Smeed, D. A. 1990 Emptying filling boxes: the fluid mechanics of natural ventilation. J. Fluid Mech. 212, 309-335.

Turner, J. S. 1973 Buoyancy Effects in Fluids. Cambridge University Press. 